\title{
Hysteresis Modeling for Magnetic Shape Memory Alloy Actuator Based on Dynamic Fuzzy Neural Network
}

\begin{abstract}
Miaolei Zhou ${ }^{a}$, Chen Zhang ${ }^{a}$, YeWei Yu ${ }^{a}$ And Shouchun Wang ${ }^{b, *}$
${ }^{a}$ Department of Control Science and Engineering, Jilin University, Changchun 130022, P.R. China

${ }^{b}$ Department of Neurology, The First Bethune Hospital of Jilin University, Changchun 130022, P.R. China

Magnetic shape memory alloy is a new class of smart metallic materials, which has excellent characteristics of huge strain and fast response. These characteristics make magnetic shape memory alloy-based actuator a potential alternative to replace traditional actuators in the high-precision positioning applications. However, the magnetic shape memory alloy-based actuator has not found its way into micro positioning field due to the obvious hysteresis behavior. In this paper, we present the prototype of the magnetic shape memory alloy-based actuator, and analyze the complex hysteresis nonlinearity between the input signal and output displacement. Then, dynamic fuzzy neural network is first utilized to construct hysteresis model for the magnetic shape memory alloy-based actuator. Dynamic fuzzy neural network is a fuzzy-logic based neural network system, which has the capability of approximating nonlinear mapping and self-adjustment. Experimental results confirm the effectiveness of the proposed hysteresis model.
\end{abstract}

DOI: 10.12693/APhysPolA.137.660

PACS/topics: hysteresis nonlinearity, magnetic shape memory alloy-based actuator, dynamic fuzzy neural network

\section{Introduction}

Recently, the intelligent materials are widely utilized in modern manufacturing, among which magnetic shape memory alloy (MSMA)-based actuator has a promising prospect in the field of nanopositioning. However, MSMA-based actuator suffers from the inherent hysteresis nonlinearity, which is a phenomenon between the input signal and output displacement, and impedes its further application [1]. Some scholars have made explorations about MSMA-based actuator. Visone et al. [2] established the Preisach model for the MSMA-based actuator. KP model based on Elman neural network was suggested in [3] to describe the asymmetric hysteresis loops. For other intelligent material-based actuators, various modeling methods have been developed [4]. However, there are still few niche modeling approaches to describe the hysteresis behaviors (amplitude-dependent and rate-dependent) in the MSMA-based actuator.

In this paper, dynamic fuzzy neural network (D-FNN) [5] is employed to describe the hysteresis in the MSMA-based actuator due to its excellent approximating ability. D-FNN can adjust its parameters and structure simultaneously via the learning algorithm developed in [6], hence the over training phenomena in the modeling process can be avoided. Experimental results show that D-FNN model has prominent performance to describe the hysteresis of MSMA-based actuator.

*corresponding author; e-mail: wangsc13@163.com

\section{Experimental characteristics of the MSMA-based actuator}

The hysteresis dynamics of MSMA-based actuator is related to the frequency of its applied input, that is, the dynamical behavior has a noticeable variation when the input frequency is changing. This is an inherent property called rate-dependent behaviour, as shown in Fig. 1a. Furthermore, the output of MSMA-based actuator depends on not only the current value but also on the previous dominant extremum of input, which results in the amplitude-dependent behaviour, as shown in Fig. 1b.

\section{D-FNN hysteresis modeling for the MSMA-based actuator}

The input-output relationship of the MSMA-based actuator is expressed as

$y_{p}(k)=\Gamma\left(u(k), \ldots u\left(k-n_{u}\right), y_{p}(k-1), \ldots y_{p}\left(k-n_{y}\right)\right)$,

where $y_{p}(k)$ and $u(k)$ are the displacement and input currents of the MSMA-based actuator, and $n_{y}$ and $n_{u}$ are the corresponding maximum lags of $y_{p}(k)$ and $u(k) . \Gamma(\cdot)$ represents the nonlinear function. For the sake of convenience, the input signal can be rewritten as $\boldsymbol{Z}(k)=$ $\left[u(k), \ldots u\left(k-n_{u}\right), y_{p}(k-1), \ldots y_{p}\left(k-n_{y}\right)\right]=\left[z_{1}, \ldots z_{j}\right]$, $j=1, \ldots p . p=n_{u}+n_{y}+1$ is the total number of input variables. Due to its remarkable approximation ability, D-FNN is used to model the nonlinear function $\Gamma(\cdot)$, and the architecture is depicted in Fig. 2. The output of the $\mathrm{D}-\mathrm{FNN}$ is given as 

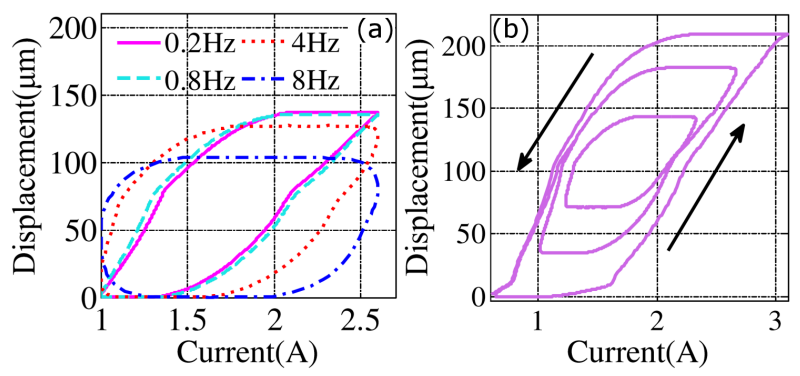

Fig. 1. Hysteresis nonlinearity of the MSMA-based actuator: (a) rate-dependent behavior, (b) amplitudedependent behavior.

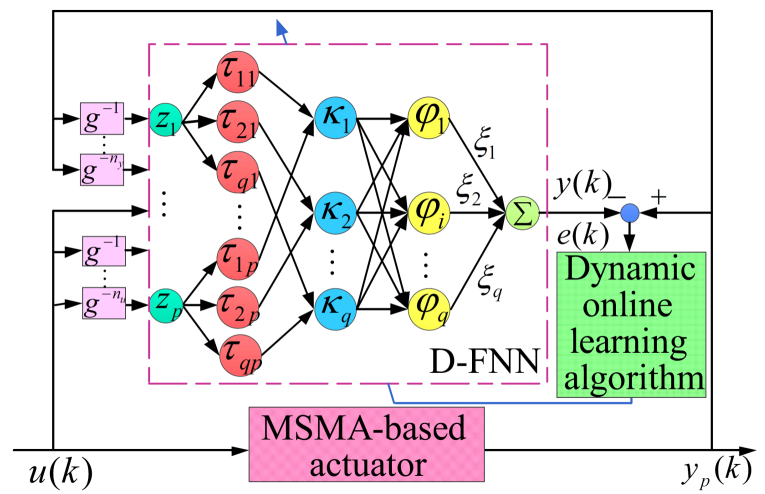

Fig. 2. Architecture of the D-FNN model.

$$
y(\boldsymbol{Z})=\sum_{i=1}^{q} \xi_{i} \varphi_{i},
$$

where $q$ is the number of rules, $\xi_{i}$ denotes the weight of each rule, and $\varphi_{i}=\frac{\kappa_{i}}{\sum_{i=1}^{q} \kappa_{i}}$ is the normalized degree of fulfillment. The Gaussian function $\tau_{j i}=\exp \left[-\frac{c\left(z_{j}-c_{j i}\right)^{2}}{\sigma_{j i}^{2}}\right]$ is selected as membership function, and the output of $i$ th rule is

$$
\kappa_{i}=\exp \left(-\frac{\sum_{j=1}^{p}\left(z_{j}-c_{j i}\right)^{2}}{\sigma_{j i}^{2}}\right)=\exp \left(-\frac{\left\|\boldsymbol{Z}-\boldsymbol{C}_{i}\right\|^{2}}{\sigma_{j i}^{2}}\right),
$$

where $\sigma_{j i}$ and $\boldsymbol{C}_{i}=\left[c_{1 i}, \ldots c_{p i}\right]$ are the width and center of Gaussian function in the $i$-th rule, respectively.

The dynamic learning algorithm for D-FNN is composed of four parts: criteria of rule generation, parameters allocation of membership function, weight adjustment, and pruning technology. Specifically, the rule generation is decided by $\|e(k)\|$ and $d_{\text {min }}$, and they are defined as:

$$
\left\{\begin{array}{l}
d_{\min }=\arg \min \left(\left\|\boldsymbol{Z}(k)-\boldsymbol{C}_{i}\right\|\right) \\
\|e(k)\|=\left\|y_{p}(k)-y(k)\right\|
\end{array}\right.
$$

For the $k$-th data $(\boldsymbol{Z}(k), y(k))$, only in the case of $\left\{\|e(k)\|>\max \left[e_{\max } \times \beta^{k}, e_{\min }\right], d_{\min }>\max \left[d_{\max } \times\right.\right.$ $\left.\left.\gamma^{k}, d_{\min }\right]\right\}$, the system will add a rule automatically. $e_{\max }, e_{\min }, d_{\max }, d_{\min }, \beta, \gamma$ are the predefined constants. Parameters of Gaussian function is decided by $\sigma_{j i}(k)=k_{w} \times \sigma_{j i}(k-1)$ and $k_{w}$ is a constant. The weight adjustment and pruning technology are achieved by recursive least squares (RLS) and error reduction ratio (ERR) methods, respectively.

\section{Experimental results}

Hysteresis experiments of MSMA-based actuator are carried out on the experimental platform shown in Fig. 3a. An exciting signal $u$ is fed into the power through PCI-1716, and the signal $u_{a}$ from this power is yielded to drive the MSMA-based actuator. Then the displacement $y_{a}$ is measured by linear variable differential transformer (LVDT), the obtained signal $y$ is transferred to host computer via PCI-1716. The configuration is shown in Fig. 3b. The MSMA-based actuator (MSM 20-E, ETO GRUPPE company) consists of MSMA element, a force spring, coils, and a push-rod. The MSMA element in the MSMA-based actuator is a NiMnGa alloy and the dimension is $14 \mathrm{~mm} \times 3 \mathrm{~mm} \times 2 \mathrm{~mm}$. The exciting signal $u_{a}$ is applied to the coils in the MSMA-based actuator and the magnetic field is obtained. The hysteresis effect is generated by this field. The specifications of LVDT and power are MDSL-0500M6-1A and PSW30-36, respectively.

Some basic parameters for constructing the D-FNN model are chosen as $d_{\max }=100, d_{\min }=0.2, e_{\max }=0.1$, $e_{\min }=0.02, \beta=0.9, \gamma=0.9, k_{w}=1.1, n_{u}=1$, and $n_{y}=1$. With this model structure, the MSMAbased actuator is fed with an exciting sinusoid signal of $u(t)=1.2 \sin (2 \pi f t)+2.2$ (frequency $f=0.2,2$, $6 \mathrm{~Hz}$ ) with different amplitudes. The modeling performance is shown in Fig. 4. It is obvious that the

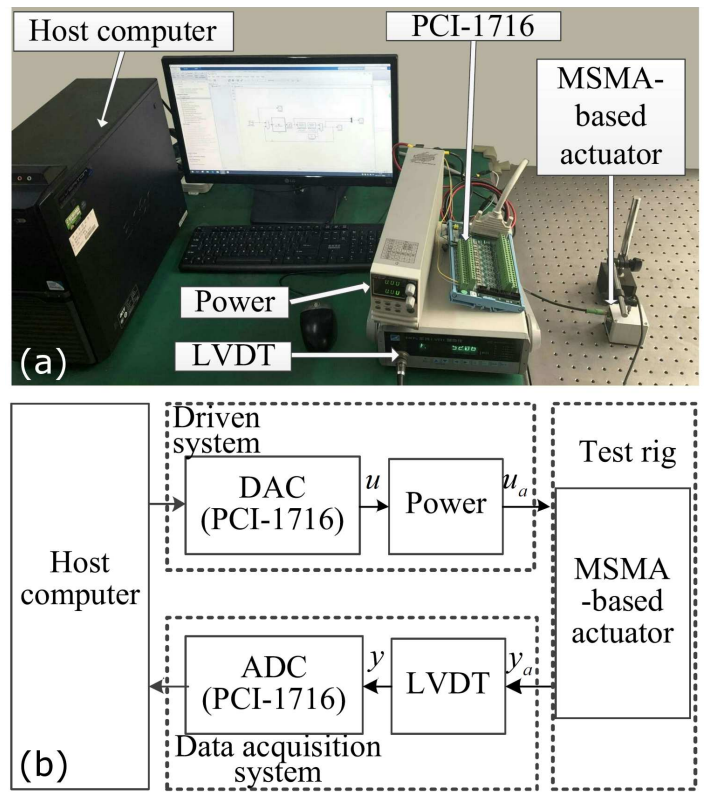

Fig. 3. Experimental platform of MSMA-based actuator: (a) picture, (b) configuration. 

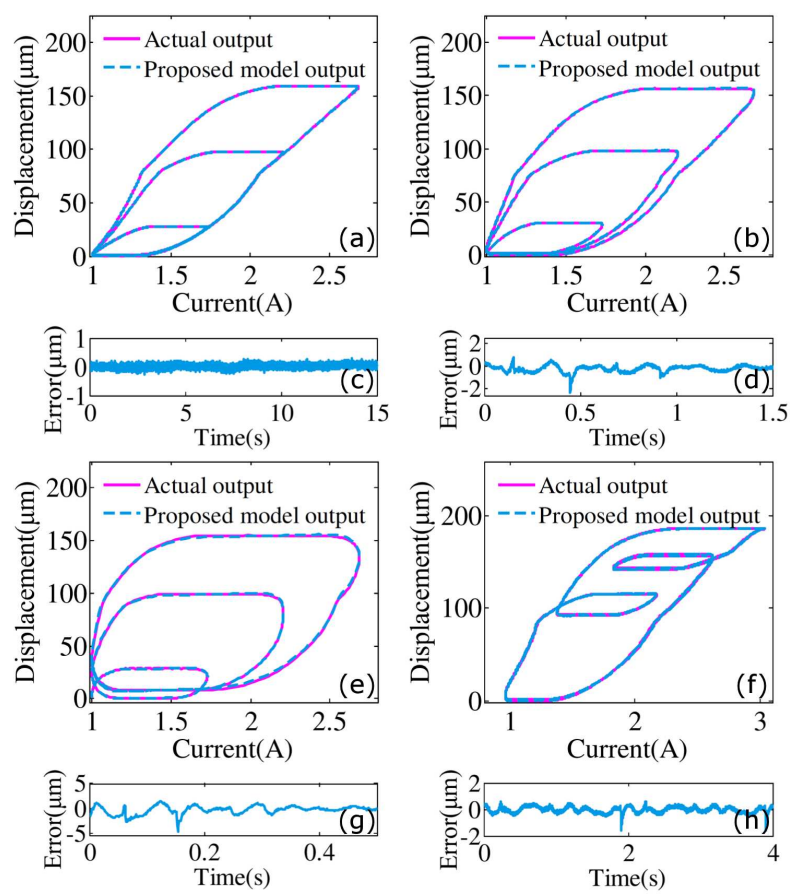

Fig. 4. Modeling performances of the D-FNN model with $0.2 \mathrm{~Hz}$ sinusoid exciting signal are shown in (a) and (c), with $2 \mathrm{~Hz}$ in (b) and (d), with $6 \mathrm{~Hz}$ in (e) and $(\mathrm{g})$, and with mixed signal in (f) and (h), respectively.

rate-dependent and amplitude-dependent characteristics of MSMA-based actuator are inherently implemented by the proposed method. More precisely, the root mean square (RMS) errors at $f \in\{0.2,2,6\} \mathrm{Hz}$ are $\{0.0714,0.3713,0.8932\} \mu \mathrm{m}$, respectively, which indicate that the model has a good ability to fit the hysteresis behavior of the MSMA-based actuator. To establish further evidences of the availability and validity of the proposed approach, a mixed signal is applied to excite the MSMAbased actuator. As described in Fig. 4f and h, the RMS error is $0.2352 \mu \mathrm{m}$ and the D-FNN model can reflect the hysteresis behavior.

\section{Conclusion}

In this paper, a D-FNN model is proposed to characterize the hysteresis nonlinearity in the MSMA-based actuator. Then the theory and structure of the D-FNN model are illustrated. A series of experiments are conducted to verify the effectiveness of the D-FNN model for describing the complex hysteresis behaviors. When the input signal is different, the RMS error can satisfy the requirement of modeling accuracy. Experimental results demonstrate that the developed approach is feasible and superior for hysteresis modeling.

\section{Acknowledgments}

This work was supported in part by the National Natural Science Foundation of China under Grant 51675228 and the Program of Science and Technology Development Plan of Jilin Province of China under Grants 20180101052JC, 20190303020SF.

\section{References}

[1] O. Heczko, V. Bradshaw, Acta Phys. Pol. A 131, 1063 (2017).

[2] C. Visone, D. Davino, A. Adly, IEEE Trans. Magn. 46, 1848 (2010).

[3] R. Xu, M. Zhou, IEEE Trans. Magn. 53, 2002004 (2017).

[4] Y. Yu, C. Zhang, M. Zhou, IEEE Trans. Nanotechnol. 19, 19310051 (2020).

[5] J. Zhai, J. Zhou, L. Zhang, W. Hong, IEEE Microw. Wirelsee Compon. Lett. 20, 528 (2010).

[6] S. Wu, M. Er, IEEE Trans. Systems Man Cybernet. Part B 30, 358 (2000). 